\title{
UNA PROPUESTA PARA LA INTRODUCCIÓN DEL CONCEPTO DE ENERGIA EN EL BACHILLERATO
}

\author{
HIERREZUELO MORENO, J. y MOLINA GONZÁLEZ, E. \\ I.B. Jorge Guillén, Torrox. \\ I.B. Reyes Católicos, Vélez-Málaga.
}

\section{SUMMARY}

This paper settles the foundations of a proposal for the introduction of the concept of energy in secondary teaching and analyses the results obtained.

La importancia del concepto de energía explica el interés que despierta su enseñanza, de lo que puede darnos una idea la gran cantidad de trabajos sobre el mismo, aparecidos en las revistas de investigación en didáctica de las ciencias (Hierrezuelo 1986).

Podemos encontrar dos posturas fundamentales sobre cómo introducir el concepto. Una, que podemos considerar mayoritaria, partidaria de definir la energía como la capacidad de un sistema para realizar trabajo. De acuerdo con lo que Warren (1982) llama punto de vista conceptualista, la energía es una idea abstracta inventada por los científicos para que les ayude en la inves. tigación cuantitativa de los fenómenos. La energía, como concepto, es algo que nada tiene que ver con la experiencia diaria, aunque la palabra se utiliza a menudo, casi siempre de manera imprecisa y con un significado ambiguo. Es un concepto que debe ser enseñado sistemáticamente, y sólo puede ser aprendido después de algunos conceptos básicos, particularmente los de fuerza y trabajo. Por lo tanto no debe enseñarse en los niveles elementales.

Otros autores se muestran disconformes con la pretensión de dar una definición operacional de la energía. Entre otros, López Rupérez y otros (1983) consideran que se debe partir de una definición descriptiva de la energía, a la que mediante un proceso gradual se le incorporen nuevos atributos hasta completar el significado del concepto. Se trata de propiciar un aprendizaje significativo por inclusión, en el sentido ausubeliano del término. Sólo al concluir este proceso didáctico tiene sentido introducir el trabajo como una medida de la energía transferida en un tipo particular de proceso.
A estos autores, y nosotros compartimos su opinión, les parece bien una definición descriptiva de la energía en los términos siguientes: "La energía es una propiedad o atributo de todo cuerpo o sistema material en virtud de la cual éste puede transformarse, modificando su situación o estado, así como actuar sobre otros originando en ellos procesos de transformación".

En un modelo generativo deI aprendizaje (Osborne y Wittrock 1985) las ideas previas de los alumnos juegan un papel fundamental. Las ideas previas sobre la energía descritas en Duit (1983) indican que los estudiantes asocian energía con fuerza, combustible, o con ciertos fenómenos como luz, sonido, etc. Watts (1983) señala algunas de las características de estas ideas de los alumnos: la energía se centra en el hombre, la confusión đe la energía con los fenómenos mismos, considerar solo la energía relacionada con el movimiento, etc.

Otros trabajos señalan la dificultad que tienen los alumnos para utilizar el principio de conservación de la energía (en adelante PCE), (Duit 1984, Driver y Warrington 1985, Solomon 1985). Duit (1985) propone la introducción de la noción de degradación de la energía, para que así el alumno pueda explicar la contradicción aparente entre lo que el lenguaje cotidiano sugiere: "consumo" o "producción" de la energía, y el principio de conservación.

\section{OBJETIVOS DE NUESTRO TRABAJO}

En primer lugar, queremos analizar y describir las ideas que sobre el significado del término energía 
tienen los alumnos, antes de recibir enseñanza formal sobre el tema. Los datos los hemos obtenido a partir de entrevistas orales y de un cuestionario escrito. Teniendo en cuenta que esas ideas provienen fundamentalmente del significado que tiene el término en el lenguaje cotidiano, pensamos que serán similares a las que ya han sido descritas en la bibliografía, aunque es posible que aparezcan algunas diferencias causadas por el idioma.

Dada la controversia existente entre las dos formas de introducir el concepto a la que hemos hecho referencia en la introducción, consideramos conveniente hacer una comparación entre los resultados obtenidos según los dos enfoques. Con este fin y dado que la opción mayoritaria entre los profesores es la de definir primero el trabajo, nosotros hemos elaborado unos materiales que creemos que pueden ser representativos de la otra tendencia en conflicto.

Las características más destacables de estos materiales son:

1) Se introduce en primer Iugar el concepto energía, mediante una definición similar a la que hemos descrito en la introducción. El concepto trabajo se introduce al final del tema, como una medida de los intercambios energéticos en unos procesos determinados.

2) Se procura la diferenciación precisa de los conceptos fuerza y energía, confundidos frecuentemente en las ideas previas de los aiumnos.

3) Se introduce la noción de degradación de la energía (Duit 1985), para explicar la contradicción entre el principio de conservación y el uso cotidiano del término.

4) Se hace un tratamiento globalizado del térnnino energía no restringiéndolo al campo de la mecánica, utilizando el concepto de energía química de los combustibles y de los alimentos.

5) Se analizan situaciones no ideales, teniendo en cuenta el efecto del rozamiento en los balances energéticos que se llevan a cabo. En cuanto a la formulación del PCE, hemos tenido en cuenta la sugerencia de Solomon (1985) de no proponer una formulación del mismo únicamente en sentido negativo "...la energía ni se crea ni se destruye, sólo se transforma...". Ha sido sustituido por una expresión en la que se alude expresamente a que se conserva la cantidad total de energía antes y después de la transformación. La definición dada ha sido: "En todas las transformaciones energéticas que ocurren en un sistema aisiado, cambia la forma en la que se presenta la energía pero no cambia la cantidad total de energía, es decir, la energía antes de la transformación es la misma que hay después de la transformación".

Nuestro segundo objetivo es comprobar si hay o no diferencias entre los resultados obtenidos por los alumnos que han seguido uno u otro método. Esto nos Heva a proponer la hipótesis, formulada en forma de hipóte- sis nula: No habrá diferencia significativa en los resul. tados de aprendizaje entre los alumnos que sigan uno y otro enfoque.

Para hacer operativa la hipótesis pretendemos contrastar los resultados de aprendizaje en los siguientes aspectos concretos:

a) Capacidad para đescribir las transformaciones energéticas que tienen lugar en un fenómeno.

b) Capacidad para diferenciar correctamente los términos fuerza y energía.

c) Capacidad para diferenciar los términos energía, trabajo y potencia.

d) Capacidad para utilizar correctamente el PCE; así como para calcular las variaciones de energía que ocurren en procesos simples.

\section{MUESTRA Y DISEÑO EXPERIMENTAL}

La muestra estaba constituida por 413 alumnos de segundo de BUP, de los cuales 217 siguieron las actividades de enseñanza diseñadas según se ha descrito y que constituyen la submuestra que Ilamaremos Experimental (exp), y los 196 restantes siguieron una ense. ñanza que tenía como rasgo distintivo el hecho de introducir en primer lugar el concepto de trabajo y posteriormente la energía definida como la capacidad de realizar un trabajo. Estos alumnos los llamaremos de control. Tanto los alumnos exp como los de control estaban distribuidos en 7 grupos con 5 profesores diferentes.

Los resultados han sido obtenidos del anátisis de una prueba escrita que se pasó a todos los alumnos. Para evitar que las respuestas estén influidas por la memorización mecánica, la prueba fue pasađa al menos 1 mes después de haber terminado los alumnos el tema correspondiente de segundo de BUP.

Para evitar el efecto Hardtone, los alumnos que siguieron el enfoque experimental no fueron informados a priori de que los resultados servirían para ser contrastados con los de los grupos de control. La metodología seguida con ellos es la misma que se sigue en los otros temas del curso, con el mismo ritmo de trabajo.

\section{ANÁLISIS DE LAS IDEAS PREVIAS DE LOS ALUMNOS}

Como ya hemos señalado, en el diseño de nuestros materiales didácticos juega un papel fundamental las ideas previas de los alumnos. Basándonos en los datos aportados por Duit (1983), Watts (1983), Driver y Warrington (1985) hemos realizado una serie de entrevistas y cuestionarios escritos para analizar el significado que para los alumnos tiene el término energía y otros términos relacionados con él, como son el de fuerza y el de trabajo. 
El cuestionario escrito se pasó a una amplia muestra de alumnos de segundo de BUP en el curso 85-86. Fue similar al utilizado por Duit (1983) y en él observamos que los alumnos asociaban el término energía con fuerza, con calor, con combustible, con Sol, con electricidad, con movimiento y con energía atómica.

Durante el curso 86-87, antes del comienzo del tema en el que se incluían estos conceptos, realizamos 31 entrevistas, de las cuales 23 fueron a alumnos de segundo y 8 a alumnos de tercero de BUP, con la intención de analizar con más detalle estas ideas. Los alumnos tenían que describir el funcionamiento de un motor eléctrico que elevaba una pesa y a continuación se les hacía una serie de preguntas relacionadas con el término energía. Fueron grabadas y posteriormente transcritas. Los aspectos más sobresalientes observađos son:

Los alumnos tienden a describir el fenómeno observado sin utilizar el término energía, a pesar de que se les animaba a que emplearan palabras científicas que creyeran que estaban relacionadas con el mismo. Sólo 4 de Ios 23 alumnos de segundo y 2 de tos 8 de tercero usaron la palabra energía en su descripción. La mayoría describe únicamente lo que ve sin dar ninguna interpretación: ". . al enchufar los cables se mueve el motor...", algunos hablan de polo positivo y negativo e incluso de electrones, si bien de una forma no coherente. Algunos añađen que el motor tendrá alguna fuerza para trabajar, ejemplo de la sustitución de la palabra energía por la de fuerza.

Cuando los alumnos no mencionaban la energía en la descripción del proceso, se les preguntaba si la energía intervenía en el mismo. Ante esta pregunta los alumnos de segundo se referían únicamente a la energía eléctrica o a la electricidad, utilizando ambas palabras de forma indistinta; los alumnos de tercero, que ya habían recibido enseñanza sobre este tema en el curso anterior, sí describían las transformaciones energéticas que tenían lugar.

A la pregunta, ¿qué significa para ti la palabra energía?, la mayoría dice que la energía es como una fuerza, "...la capacidad de fuerza que tiene un cuerpo...". Esta asociación fuerza-energía se muestra como una de las ideas previas más persistentes en estos alumnos. Otro grupo también numeroso la asociaba con el movimiento, en concreto con algo capaz de moverse por sí mismo. En este sentido, las personas tienen energía porque son capaces de moverse, la pesa que era elevada por el motor no tiene energía porque no puede moverse por sí misma. Algún alumno señalaba que la pesa sólo tiene energía cuando está subiendo.

Entre los alumnos de tercero se observa que identifican con más facilidad la energía cinética que tiene la pesa cuando sube que la ganancia de energía potencial que experimenta en la subida. El concepto de energía potencial parece mucho menos evidente que el de energía cinética; los alumnos que aún no han recibido enseñan$z a$, no reconocen que un cuerpo en una posición elevada tenga energía debido a esa posición: "...la pesa en lo alto del armario no tiene energía...", mientras que muchos asocian una energía a los cuerpos en movimiento.

Al preguntarles por otros cuerpos que tuvieran energía, de los presentes en el local donde se realizaba la entrevista, la mayoría se refería a la energía eléctrica (localizada en el radiocasette, en los focos de luz, enchufes, etc.) y prácticamente todos señalaban las personas, entrevistador y entrevistado, como poseedoras de energía. Sin embargo, los objetos inanimados y en reposo, como la mesa o un cuerpo en lo alto de un armatio, no poseen energía de ningún tipo. Los alumnos dicen que las personas tienen capacidad para moverse por sí mismas, de ejercer fuerzas, mientras que los objetos inanimados no la tienen siendo ésta la razón que dan para decir si un cuerpo tiene o no energía.

Se observa en las entrevistas que los alumnos utilizan indistintamente los términos de fuerza, potencia, trabajo y energía en la descripción de una situación: “...algo tiene energía porque es potente y es potente porque ejerce o tiene fuerza...". Siendo fundamental marcar la diferencia que existe entre la precisión del lenguaje científico y la ambiguiedad del lenguaje cotidiano, será necesario tener este hecho en cuenta al programar la enseñanza de estos conceptos.

En cuanto al PCE, no es usado por los alumnos de segundo de BUP. La mayoría dice que un futbolista tiene menos energía después de haber jugado un partido que al principio, y cuando se le pregunta qué ha pasado con esa energía dicen que se ha gastado, que se ha perdido, que ha desaparecido. De manera similar, cuando se refieren a la recuperación de esa energía, puede hacerse por dos caminos diferentes: o bien tomando alimentos o simplemente "descansando". Es decir, podemos aumentar de energía sin tomarla de ninguna parte.

En alumnos de tercero de BUP se observa una coexistencia de las ideas previas con lo aprendido sobre el PCE. Así dicen que el cigarro "produce" energía calorífica, aunque hayan explicitado varias veces durante la entrevista que la energía se transforma pero no se pierde.

\section{RESULTADOS Y ANÁLISIS DE RESPUESTAS}

Los resultados se refieren a las respuestas de los alumnos a la prueba que incluimos en el anexo. Por razones de espacio, sólo analizaremos algunas preguntas. Los datos estăn siempre expresados en porcentajes y NC se refiere a los alumnos que no contestan.

\section{Pregunta 1:}

Se han clasificado las respuestas según que la altura señalada fuese correcta o incorrecta y atendiendo al tipo de explicación que justifica la opción escogida. El significado de cada una de las categorías es:

B1: Apoyan su respuesta en el PCE y en las transformaciones energéticas que tienen lugar. Incluimos tanto 
los que mencionan el rozamiento como fenómeno que puede hacer variar la altura a la que puede subir, como aquéllos que no lo hacen, que suponen implícitamente una situación ideal.

B2: Predicen la altura correcta, no utilizando el PCE para justificar su respuesta.

M: Aquéllos que predicen una altura incorrecta o dan una explicación errónea.

Tabla I

Clasificación de respuestas en la pregunta 1 Experimental Control

$81 \quad 36,7 \quad 16,3$

B $24,3 \quad 30,6$

M $\quad 38,5 \quad 49,5$

$\mathrm{NC} \quad 0,5 \quad 3,6$

Ya habiamos observado en las entrevistas tealizadas para detectar las ideas previas, el poco uso que hacen los alumnos del término energía para explicar los fenómenos. Tras la enseñanza, los resultados están en esa misma dirección, aunque los alumnos exp utilizan el PCE en una proporción muy superior a los de Control. Estos datos coinciden con los señalados por Duit (1984) y Driver y Warrington (1985).

Un aspecto destacable es que los alumnos de control no mencionan el rozamiento en sus respuestas, sólo lo hace el $4,6 \%$, mientras que los exp se plantean explícitamente las dos posibilidades, que exista o no rozamiento, en un porcentaje significativamente más alto, el $34,4 \%$.

Entre las respuestas incorrectas hemos observado que un porcentaje alto de los alumnos exp describen las transformaciones energéticas que sufre la bola, aunque no utilizan el PCE. Por el contrario, los de Control, ni siquiera emplean el término energía. Son respuestas intuitivas, sin explicación lógica alguna. En estas respuestas se detectan algunos errores conceptuales como el hecho de asignar la magnitud fuerza a la bola como una propiedad de la misma, (fuerza de los objetos, Viennot 1979), o confundir velocidad con fuerza: "...la bola va perdiendo fuerza al subir..." "...subirá segün la fuerza que lleve...", "...la fuerza que coge bajando...". Otro error común es la confusión entre fuerza y energía: "...por el rozamiento pierde fuerza...".

\section{Pregunta 2:}

B: Aquéllas que identifican la necesidad de energía cuando hay una transformación y señalan que no es necesario energía cuando permanece el mismo estado. Este criterio es válido para los tres ítems de la pregunta.

MI: Los que dan una explicación dinámica, siendo ésta correcta, pero no relacionan la necesidad o no de energía con la existencia de una transformación. En el ítem 2.3, al no existir esa posibilidad, hemos incluido todas las respuestas incorrectas.

M2: En los ítems 2.1 y 2.2 las respuestas incorrectas desde cualquier punto de vista.

Tabla II:

Clasificación de respuestas en la pregunta 2

\begin{tabular}{cccccccc} 
& \multicolumn{1}{c}{} & \multicolumn{2}{c}{ Experimentales } & & \multicolumn{3}{c}{ Control } \\
& 2.2 & 2.2 & 2.3 & & 2.1 & 2.2 & 2.3 \\
B & 65,1 & 51,4 & 18,8 & & 28,1 & 5,1 & 3,5 \\
M1 & 1,4 & 9,2 & 77,1 & & 25,5 & 55,5 & 82,7 \\
M2 & 30,8 & 36,7 & & & 37,8 & 32,7 & \\
NC & 2,8 & 2,7 & 4,1 & & 8,7 & 10,7 & 13,8
\end{tabular}

Con esta pregunta pretendiamos comprobar si el alumno diferencia entre Io que significa que en un estado exista energía y lo que significa que en un proceso haya una variación de energía. Los datos recogidos en la tabla II son elocuentes en el reflejo de la diferencia entre las respuestas de ambas muestras. Entre los alumnos de Control, destaca el elevado porcentaje que hace uso de justificaciones dinámicas, aunque se les preguntaba explf́citamente por la energía.

Merecen destacarse los resultados del ítem 2.3 con un porcentaje de respuestas incorrectas muy elevado. Muchos alumnos indican que se necesita una fuerza para mantener estirado el muelle y por lo tanto se necesita una energía, lo que consideramos un ejemplo de la persistencia de la confusión entre fuerza y energía. Otros, comentan que un muelle estirado tiene energía, confundiendo el que exista energía con la necesidad de tranferencia de energía para mantener un determinado estado.

Una idea errónea que se repite con frecuencia es la necesidad de la existencia de atmósfera para que exista o para que actue la gravedad. Así indican que: ...en ese planeta el tren flotaría porque no hay atmósfera, y por lo tanto no se necesita energía...". Algo similar fue observado por Ruggiero y otros (1985).

Algunos alumnos señalan que al muelie, en el ítem 2.3, no hay que darle energía porque no está en movimiento. La asociación de la energía con el movimiento es también frecuente entre los alumnos.

\section{Pregunta 4:}

En la misma debían utilizar, al menos una vez, cada uno de los siguientes términos: fuerza (4.1), energía (4.2), trabajo (4.3) y potencia (4.4). Los números entre paréntesis identifican el término que le precede.

Hemos considerado correcta la respuesta sobre fuerza cuando la expresaban de alguna manera como interacción entre dos cuerpos: "...el carro al chocar ejerce una fuerza sobre el tope...". Respecto a la energía cuando mencionan la energía cinética o potencial o 
alguna de las transformaciones que tienen lugar: “...el carro lleva una energía cinética debido a su velocidad..."; "..el cuerpo que sube aumenta su energía potencial...". Respecto al trabajo si lo relacionan con una fuerza y un desplazamiento o si lo mencionan como medida đe una transformación energética. Respecto a la potencia si la refieren como energía transformada o trabajo realizado en ia unidad de tiempo.

Tabia III

Clasificacion de respuestas en la pregunta 4

\begin{tabular}{|c|c|c|c|c|c|c|c|c|}
\hline & \multicolumn{4}{|c|}{ Experimental } & \multicolumn{4}{|c|}{ Control } \\
\hline & $4 J$ & 4.2 & $\underline{4.3}$ & $\underline{4.4}$ & 4.1 & 4.2 & $\underline{4.3}$ & 44 \\
\hline B & 65,1 & 75,7 & 58,7 & 32,7 & 18,4 & 17,8 & 12,2 & 8,1 \\
\hline M & 32,6 & 20,6 & 37,6 & 62,3 & 56,1 & 58,7 & 65,9 & 64,3 \\
\hline $\mathrm{VC}$ & 2,3 & 3,7 & 3,7 & 5,0 & 25,5 & 23,5 & 21,9 & 27,6 \\
\hline
\end{tabular}

Existe una diferencia cualitativa entre las respuestas de ambos grupos reflejada en la extensión y coherencia de las mismas. En los alumnos de control, son más reducidas y están menos relacionadas con el proceso que tienen que explicar, siendo destacable el alto porcentaje de alumnos que no contestant. Se observa una dificultad para describir un fenómeno interpretándolo mediante conceptos científicos apropiados; en muchos casos se limitan a describir los aspectos observables, a pesar de que en el enunciado se les pedía que utilizaran los términos fuerza, energía, trabajo y potencia. Ejem. plo de ese tipo de respuesta descriptiva sería: “...el cochecito va corriendo y choca con el tope, hace que las ruedas se muevan y esto hace subir al cuerpo B...". En otras ocasiones introducen los términos que se les piden de forma forzada construyendo una frase sin sentido lógico: "...el carro lleva fuerza, da energia que produce trabajo y éste se transforma en potencia que hace que B suba...".

Entre los errores más frecuentes observados destaca la confusión fuerza-energía, que en este caso se manifiesta al decir que la fuerza se convierte en energía. Una respuesta típica sería: "...el carro lleva una determinada fuerza que se transforma en energia al contactar con el tope, entonces se produce un trabajo que hace que los engranajes se muevan y el objeto $B$ sube con una determinada potencia...". Podemos ver como se asigna una fuerza al coche, en el sentido de fuerza de los objetos que se mueven (Viennot 1979), sustituyendo ese concepto de fuerza al correspondiente de energía cinética. En otros casos la energía cinética del carro es la que ejerce la fuerza sobre el tope. No se ha producido un proceso de cambio conceptual, coexis. tiendo en la mente del alumno tanto el término fuerza como el de energía con un significado ambiguo y confuso (Posner et al, 1982).

El término potencia es utilizado confusamente, observándose la influencia del lenguaje diario. Así son contestaciones frecuentes: "...el carro va con una potencia debido a su velocidad...", "...el carro lleva una determinada potencia y al chocar se transforma en energía..." " "...la potencia que lleva el carro desarro. lla fuerzas." En pocos casos se relaciona la potencia con una transformación energética y el tiempo necesario para la misma. Es patente por lo tanto la confusión entre los conceptos de potencia y energía.

\section{Pregunta 6:}

En la pregunta 6.1 hemos clasificado como correctas aquellas respuestas que escogen la opción D y señalan que los cuerpos no tienen fuerza. En la pregunta 6.2 consideramos correctas aquellas respuestas que hacen uso del principio de conservación de la energía (PCE).

Tabla IV

Clasificación de respuestas en la pregunta 6

\begin{tabular}{|c|c|c|c|c|}
\hline \multicolumn{3}{|c|}{ Experimentales } & \multicolumn{2}{|c|}{ Control } \\
\hline & $\underline{6.1}$ & 6.2 & 6.1 & 6.2 \\
\hline B & 49,1 & 61,4 & 1,5 & 14,1 \\
\hline $\mathrm{M}$ & 50,4 & 37,7 & 92,9 & 78,1 \\
\hline $\mathrm{NC}$ & 0,5 & 0,9 & 5,6 & 7,7 \\
\hline
\end{tabular}

En el f́tem 6.l vemos como se mantiene en los alumnos de control la idea ya señalada de que los cuerpos en movimiento tienen una fuerza que no existe cuando esos cuerpos están en reposo. Aproximadamente el $90 \%$ consideraron que el coche tiene más fuerza en la posición 1 que en la 2 porque está en movimiento, porque lleva velocidad o por cualquier otra razón similar. Los resultados de los alumnos exp son mucho mejores con cerca de un $50 \%$ de respuestas correctas.

En cuanto al ítem 6.2 cuya contestación correcta supone la aplicación del PCE, la situación es similar siendo muy pocos los alumnos de control que responden adecuadamente. Dentro de las respuestas correctas, se observa que un $20 \%$ del total de la muestra de alumnos exp hace alusión a una situación real con rozamiento, explicando el efecto del mismo; sin embargo, entre los alumnos de control, son muy pocos los que se refieren a la situación real, sólo el 2,5\%, aplicando el PCE a una situación ideal sin rozamiento.

Conviene destacar algunas de las explicaciones que ofrecen para justificar sus respuestas erróneas. Así dicen frecuentemente: "...tiene más energía en 1 porque estaba moviéndose..." dejando ver una asociación energía-movimiento en el mismo sentido que ya ha sido señalado por Watts (1983). Este tipo de respuesta supone también el no tener en cuenta la energía poten. cial, que es olvidada por un grupo significativo de alumnos. De este modo, estos alumnos dicen que tiene más energía en 1: "...porque si no no habría podido subir la cuesta...", frase que refleja la necesidad de una energía para cambiar la altura pero no se considera que se teng a más energía por el hecho de haber cambiado de posición. Incluso hay algunos que escogen la opción 1 argumentando que la energía cinética es "mayor" que 
la energía potencial, cuando no hay datos que permitan avalar una afirmación de ese tipo.

Otro grupo numeroso de respuestas erróneas vuelve a poner de manifiesto la confusión fuerza-energía que ya hemos mencionado. Algunos dan la misma respuesta para ambos apartados, siendo frecuente leer respuestas del tipo: "...tiene más energía en I porque al tener más fuerza tiene más energía.." o esta otra: "...tienen la misma energía porque en ambos casos los motores están parados y por lo tanto no hay fuerza motora...".

\section{Pregunta 8:}

En este caso la clasificación la hemos hecho atendiendo a los siguientes criterios: Son correctas (B) las respuestas que indican que no creen que funcione el mecanismo, ya que según el PCE no se podría producir energía y por tanto tampoco se podría aprovechar.

Los resultados indican que los alumnos exp hacen uso del PCE en una proporción muy superior a la de los alumnos de control. Conviene señalar que a los grupos exp no se les había dicho nada sobre el movil perpetuo de primera especie, de forma que en sus respuestas no aluden nunca a esa justificación, sino que aplican el

Tabla V

Clasificación de respuestas en la pregunta 8

$\begin{array}{lcc} & \text { Experimentales } & \text { Control } \\ \text { B } & 21,6 & 6,3 \\ \text { M } & 74,3 & 67,8 \\ \text { NC } & 4,1 & 25,9\end{array}$

PCE diciendo que no es posible un suministro continuo de energía sin un aporte similar por otro lado. Entre Ios alumnos de los grupos de control hay algunos que justifican la imposibilidad por el hecho de que es un móvil perpetuo de primera especie, sin añadir ninguna otra explicación.

Entre las respuestas erróneas se observan dos grupos. Aquéllos que no están de acuerdo con el inventor pero no justifican o dan explicaciones en las que no se tiene en cuenta el PCE. De éstos, muchos dicen que no funcionaría por el rozamiento que lo haria detenerse; otros creen que no pesa más la cadena de un lado que en el otro; y una mayoría dice que no funciona porque no hay ninguna fuerza que lo haga moverse. A estos alumnos les extraña que un mecanismo tan simple pueda funcionar, además sin ninguna fuerza que lo haga moverse. El segundo grupo de respuestas erróneas lo constituyen aquéllos que están de acuerdo con la opinión del inventor, aunque algunos muestran algu* nas reticencias e indican que se podría aprovechar muy poca energía.

\section{CONCLUSIONES}

Los resultados obtenidos por los alumnos de los grupos exp han sido muy superiores a los obtenidos por los alumnos de control en todas las preguntas. Sin embargo, no nos atrevemos a asegurar que la diferencia ha sido debida únicamente a cómo se ha introducido la energía, ya que también ha sido diferente la metodología utilizada. No nos ha sido posible realizar el contraste entre las dos formas de introducir la energia utilizando la misma metodología de descubrimiento orientado. Así pues, no podemos afirmar que las diferencias se deban a la estructura del tema y no a la metodología o viceversa. Nosotros nos inclinamos porque han influido los dos factores.

Los resultados muestran que los alumnos exp tienen una mayor capacidad para describir las transformaciones energéticas que tienen lugar en un fenómeno, para diferenciar los términos fuerza y energía, trabajo y potencia y para usar el principio de conservación de la energía.

Como era previsibie, hemos observado diferencias en los resultados obtenidos por los alumnos de profesores diferentes, tanto en los exp como en los de control, incluso estas diferencias han existido entre los alumnos de dos grupos diferentes que habían recibido enseñanza con el mismo profesor. Sin embargo, en la práctica totalidad de los casos, el grupo experimental que tenía el peor resultado, superaba el mejor de los resultados obtenidos por los grupos de control. Esto nos hace pensar que las diferencias observadas no deben ser atribuibles a la influencia del profesor sino que el papel predominante lo juega el método empleado.

Del análisis de las respuestas podemos sacar las siguientes conciusiones:

1. La principal dificultad conceptual radica en la diferenciación entre fuerza y energía, más precisamente, la dificultad surge por la confusión que existe entre la fuerza de los objetos que se mueven, idea mantenida por los alumnos antes y después de la enseñanza, y la energía cinética que debe sustituir a esa idea.

Una enseñanza programada para eliminar esa confusión parece conseguixlo en un porcentaje significativo. En caso de un enseñanza que no ha hecho especial hincapié en estos aspectos, las concepciones de los alumnos permanecen inalteradas casi en su totalidad (véase resultados de la pregunta 6). Se hace necesario insistir en estos aspectos básicos en cursos posteriores de manera que pueda lograrse un cambio conceptual en un porcentaje amplio de alumnos.

2. Los alumnos muestran una preferencia por resolver los problemas dinámicamente en lugar de energéticamente. Esto es explicable porque en el currículo se dedica mucho más tiempo al estudio de la dinámica que al estudio de la energía. Además, la magnitud que el alumno tiende a utilizar espontáneamente es la fuerza en lugar de la energía. Los alumnos exp, que dedicaron muy poco tiempo al estudio de la dinámica en este 
curso, hacen uso en mayor proporción de un añálisis energético de las situaciones planteadas.

3. EI PCE juega un papel fundamental en la comprensión de lo que es la energía, tanto que algunos autores Ia definen como una magnitud abstracta que se conserva (Feynman 1971). En la prueba debían hacer uso del PCE en varios ítems. Observamos que los porcentajes de aciertos son sustancialmente diferentes entre una y otra pregunta; el resolver correctamente una pregunta depende no sólo del concepto o de la relación que han de usar, sino, y esto es importante, de la presentación de la pregunta.

Creemos que la observación que hace Solomon (1985) sobre Ia conveniencia de una formulación positiva del PCE contribuye a que los alumnos lo utilicen mejor en la resolución de problemas. Asimismo la noción de la degradación de la energía es útil para eliminar la contradiccion entre el principio de conservación y el lenguaje cotidiano.

Orro aspecto que también consideramos positivo es ampliar la aplicación del PCE a situaciones en las que intervengan otras energías diferentes a las mecánicas. Los alumnos de control sólo utilizan el PCE para igualar la energía cinética a la potencial, incluso en casos donde no es aplicable dicha igualdad. Por el contrario, no tienen en cuenta otros sistemas que intervienen en las situaciones que tienen que analizar. Estos alumnos en raras ocasiones hacen un análisis de las situaciones reales, tienden a idealizarlas, suponiendo que no hay rozamiento.

4. La confusión entre los significados de las palabras: fuerza, energía y trabajo, que los alumnos utilizan de forma incorrecta en la pregunta 4 , nos hace dudar de la eficacia de una enseñanza en la que los conceptos quedan sin delimitar y los alumnos no son capaces de

\section{REFERENCIAS BIBLIOGRÁFICAS}

BROOK, A. y DRIVER, R., 1984. Aspects of secondary students understanding of energy: full report. (Publicacion de la Universidad de Leeds).

BROOK, A. y DRIVER, R, 1986. The construction of meaning and conceptual change in classroom settings: case studies on energy. (Publicación de la Universidad de Leeds).

DRIVER, R. y WARRINGTON, L., 1985. Student's use of the principle energy conservation in problems situations Physics Education, Vol. 20, pp. 171-176.

DUIT, R, 1983. Energy conception held by students and consequences for science teaching en Misconceptions in Science and Mathematics, pp. 316-321.

DUIT, R., 1984. Learning the energy concept in schoolempirical results from the Phylippines and West Germany, Physics Education, Vol. 19, pp. 59-66.

DUIT, R., 1985. In search of an energy concept (Paper prepared for "Conference on Teaching about Energy within the Secondary School Science Curriculum", Leeds. aplicarlos a situaciones relativamente simples.

La dificultad que les supone a los alumnos la descripción de esos fenómenos simples, junto al alto número de alumnos que no contesta a estas cuestiones nos hace sospechar que en la enseñanza por ellos recibida no se han incluido actividades de este tipo. Nos parece que este es un mal que aqueja con bastante generalidad a la enseñanza de las ciencias, acusadamente en la Física y Química. Parece que es suficiente que el alumno sea capaz de definir correctamente un término y a lo sumo que sea capaz de aplicar una fórmula para calcular una determinada magnitud. Sin embargo, nos parece un aprendizaje de poca calidad, cuando no es capaz de utilizar los conceptos físicos que ha estudiado para describir una siquación física. No decimos que sean capaces de explicar un determinado fenómeno, sino que sean capaces de describirlo utilizando los términos científicos. De esta manera podemos saber si comprenden verdaderamente el significado de los mismos. Pero para esto es necesario que se programen las actividades adecuadas en las clases.

El análisis cualitativo de los fenómenos que propugnamos no está reñido con un estudio cuantitativo de los mismos. Lo que defendemos es no quedarse simplemente en los cálculos numéricos, sino que estos acompañen a la interpretación física de las situaciones propuestas.

5. Los resultados indican que no ha tenido ningún éxito la enseñanza en cuanto a Ia comprensión de que el trabajo mide una de las formas de variación de la energía de un sistema. Creemos necesario estudiar este aspecto más detalladamente, intentando discernir si ha sido causado por un mal diseño de las actividades o por tener una dificultad intrínseca tan elevada que quizás resultara más rentable programar este punto en cursos posteriores.

FEYNMAN, R. et al. 1971. Física 1. Mecánica, radiación y calor. (Fondo Educativo Interamericano: Bogotá).

GIL PEREZ, D., 1983. Tres paradigmas básicos en la enseñanza de las Ciencias, Enseñanza de las Ciencias, Vol. I, pp. 26-33.

GIL, D. y MARTÍNEZ-TORREGROSA, J., 1983. A model for problem solving in accordance with scientific methodology, European Journal of Science Education, Vol. 5 , pp. 447-455.

HIERREZUELO MORENO, I, 1986. Revisión bibliogrăfica sobre la enseñanza de la energía, Enseñanza de las Ciencias, Vol. 4, pp. 266-268.

LOPEZ RUPÉREZ, F. y LÓPEZ RUPÉREZ, E., 1983. Las nociones de trabajo y energía. Análisis conceptual y didáctico, Bordón, 249, pp. 497-506.

OSBORNE R. y WITTROCK M., 1985. A generative model and its implications for Science Education, Studies in Science Education, pp. 59-87. 
POSNER et al., 1982. Accomodation of a Scientific Conception: Toward a theory of Conceptual Change, Science Education, Vol. 66, pp. 211-227.

SOLOMON, J., 1985. Teaching the conservation of energy, Physics Education, Vol. 20, pp. 165-170.

RUGGIERO, S . et al., 1985. Weight, gravity and air pressure: Mental representation by Italian middle school pupils, European Journal of Science Education, Vol. 7, pp. 181194.

\section{ANEXO}

1.- En un experimento de laboratorio un alumno deja caer una bola de cojinete, desde el punto $X$, sobre un carril metálico. Señala cuál será el punto más lejano que puede alcanzar la bola.

\section{Explica tu respuesta.}

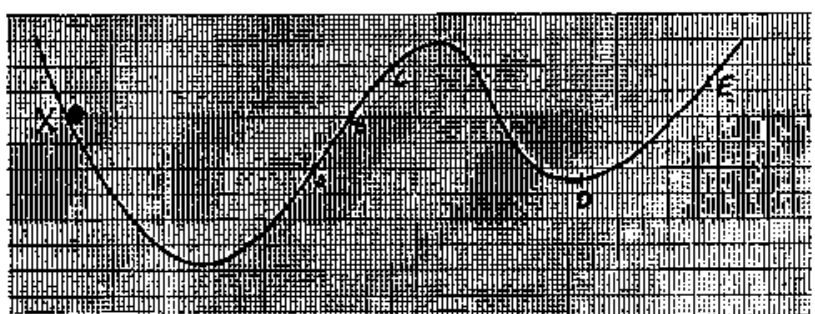

2. - Supón que estás en un planeta en el que no hay atmósfera de ningún tipo y en el que existen unos materiales que permiten el desplazamiento sin que haya rozamiento.

2.1.- ¿Será necesario usar energía para poder poner en movimiento, a una velocidad de $70 \mathrm{~km} / \mathrm{h}$, un tren cuya masa es de $10.000 \mathrm{~kg}$ ? Explica tu respuesta.

\section{2.- ¿Sera necesaria energía para que el tren se mantenga en} movimiento? Explica tu respuesta.

2.3. - ¿Será necesaria energía para mantener un muelie estirado? Explica tu respuesta.

4.- El carro representado en la figura lleva una determinada velocidad. Al chocar contra el tope es capaz, mediante un sistema de engranajes, de subir una determinada altura el peso que tenemos colocado en B.

Describe el proceso, usando al menos una vez cado una, las palabras fuerza, energía, trabajo y potencia.

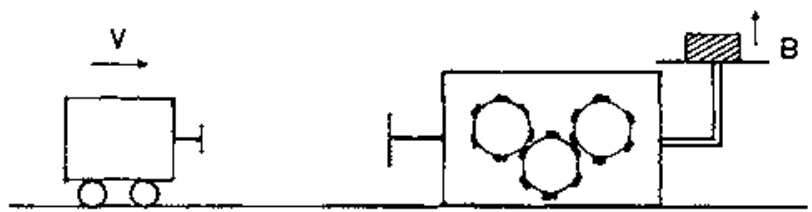

VIENNOT, L., 1979. Spontaneous reasoning in elementary dynamics, European Journal of Science Education, Vol. 1, pp. 205-211.

WARREN, J.W., 1982. The nature of energy, European Journal of Science Education, Vol, 4, pp. 295-297.

WATTS, M., 1983. Some alternative views of energy, Physics Education, Vol. 18, pp. 213-217.

6.- Un coche llega al principio de una cuesta con una determinada velocidad. En ese momento se le para el motor, pero aún puede subir hasta lo alto de la cuesta, lugar donde se detiene.

Señala qué frase te parece correcta. explicando tu respuesta: 2

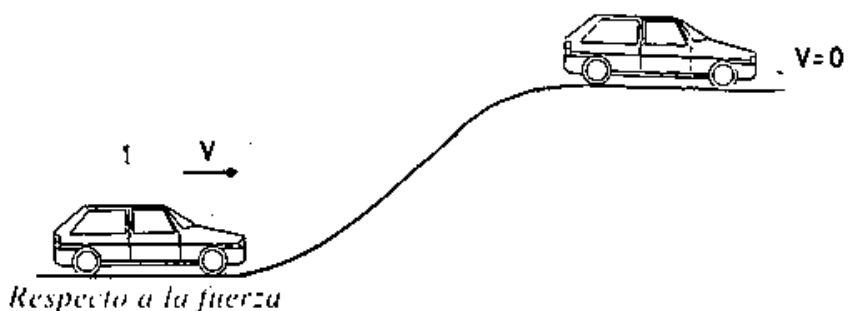

A: Tiene más fuerza en 1 .

B: Tiene más fuerza en 2.

C: Tiene la misma fuerza en I que en 2 .

D: Ninguna es correcta.

\section{Respecto a la energía}

A: Tiene más energía en 1

B: Tiene más energía en 2

C: Tiene la misma energía en 1 que en 2

D: Ninguna es correcta

8.- Observa el dispositivo de la figura. Su inventor hace la siguiente defensa del aparato.

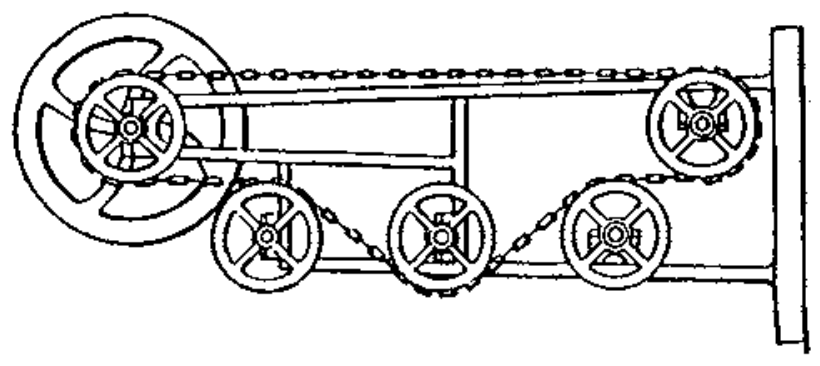

Cualquiera que sea la posición de la cadena, su lado derecho debe pesar más que el izquierdo. Por consiguiente, esta parte de la cadena debe tirar de la otra e ir bajando ininterrumpidamente, con to cual hará que se mueva todo el mecanismo. En todo ese proceso nosotros podemos ir aprovechando directamente la energía que se va produciendo"

¿Estás de acuerdo? Justifica tu opinión, añadiendo todos los comentarios que creas oportunos. 